\section{GROWTH AND PUBERTY IN THE COELIAC DISEASE OF THE CHILD}

\author{
M. Bessahraoui, K. Bouziane \\ University of Medecine, Oran, Algeria
}

The aim of the present study was to evaluate growth, celiac disease (CD) adult height and age at menarche.

\section{Subjects and methods:}

1. Growth was assessed by:-Longitudinal study, compared with Sempé, Pédron subjects.-Semi longitudinal case-control study. Controls (eliac brothers and sisters).

2. Age at menarche was assessed by case-control study. Controls ( eliac sisters).

\section{Results:}

1. Growth was significantly more delayed in $C D$ patients (103 girls,92 boys) than in Sempé, Pédron subjects. However, evident catch-up growth was noted between the 18 and 21 years age ( 103 CD girls and 92 CD boys CD).

2. The growth speed was less important during puberty compared to Sempé, Pédron subjects. After 18 age, our CD patients was contuned their growth in time where Sempé, Pédron subjects finished their growth.

3. When CD was associated with Diabete type 1 , growth was significantly more delayed than in the $\mathrm{CD}$ isolated or diabetes patients isolated.

4. Semi-longitudinal study: The mean adult height was: $158,42 \pm 6,3 \mathrm{~cm}$ ( 269 women CD) vs 162 , $17 \pm 6,3 \mathrm{~cm}$ (193 controls $: p<0,0001)$. The mean adult height was 170, $28 \pm 7,5 \mathrm{~cm}$ (194 men CD) and $172,53 \pm 6,8 \mathrm{~cm}(200$ controls: $p<0.09) .5$. The mean age of menarche in $174 \mathrm{CD}$ was: 4,56 $\pm 1,63$ years vs $13,74 \pm 1,36$ years in 174 controls $(p<$ 0,0001).

Conclusion: Growth, puberty and adult Height are delayed especially in the CD girls. Additional factors influencing growth, is the auto immuns diseases associated.

\section{THE ROLE IN CELL DEATH IN CHOLESTASIS MURINE}

\author{
M.-M. Tiao ${ }^{1}$, Y.-J. Yang ${ }^{2}$
}

${ }^{1}$ Pediatrics, Chang Gung Memorial HospitalKaohsiung Medical Center, Chang Gung University College of Medicine, Kaohsiung, ${ }^{2}$ National Cheng Kung University and Hospital, Tianan, Taiwan R.O.C.

Objective: We find that the biogenesis of mitochondria is response to the early stage of cholestasis with internal pathway. The regulation pathogenesis of the liver cell apoptosis in this early stage in cholestatic liver injury is not clear.

Methods: Arat model of cholestasis was established by bile duct ligation(BDL), with simultaneous creation of the sham group receiving laparotomy without BDL. Liver LC3B1, LC3B2, Beclin-1, caspase 9 protein expression was analyzed by western blotting. The ATF6, IRE1, BIP RNA level was measured by realtime polymerase chain reaction.

Results: The liver LC3B-2 and LC3B2/LC3B1 ratio at 6-72 hours with caspase 9 activity and Beclin-1 levels at 72 hours significant upregulated. The ATF6, IRE1, BIP significantly decreased at 24- 72 hours.

Conclusions: Our results indicate that regulation of the liver biogenesis is within a few hours after complete bile duct obstruction. Early murine cholestatic liver injury is via autophagy pathway.

763

\section{APPLICATION OF STOOL ALPHA-1 ANTITRYPSIN IN PEDIATRIC PATIENTS WITH GASTROINTESTINAL DISEASES}

\section{C.-Y. Yeung, H.-C. Lee, W.-T. Chan, C.-B. Jiang \\ Division of Gastroenterology and Nutrition, Department of Pediatrics, Mackay Memorial Hospital, Taipei, Taiwan R.O.C.}

Objectives and study: In children with gastrointestinal (GI) disease, ongoing losses of endogenous proteins have been suggested as contributing to impairment of nutritional and immunologic status. Quantification of stool alpha-1 Antitrypsin (sAAT) excretion is established for estimation of enteric protein loss and assessment of disease activity. This study is to access the 